\title{
Study of the Log-Layer Structure in Wall Turbulence Over a Very Large Range of Reynolds Number
}

\author{
Ivan Marusic • Nicholas Hutchins
}

Received: 7 March 2007 / Accepted: 27 September 2007 / Published online: 24 November 2007

(C) Springer Science + Business Media B.V. 2007

\begin{abstract}
Studies of the logarithmic layer structure in turbulent boundary layers are presented that span three orders of magnitude change in Reynolds number. The experiments considered used two separate laboratory scale facilities, as well as the atmospheric surface layer at the SLTEST facility in Utah. Several experimental techniques were used in order to probe the three-dimensional nature of the flow structures. The main focus is on two-point correlation statistics at a given $z / \delta$, which are found to agree well over all Reynolds numbers when scaled with an outer lengthscale. Large-scale coherence recently noted in the logarithmic region of laboratoryscale boundary layers is also found to be present in the atmospheric surface layer flow. Recent findings regarding the influence of these large scale motions on the nearwall region are also presented.
\end{abstract}

Keywords Wall turbulence - Logarithmic region • Superstructure • Atmospheric surface layer $\cdot$ High Reynolds number

\section{Introduction}

Recent studies [1-5] have presented clear evidence that the logarithmic region in wall turbulence (defined here tentatively as $z^{+}>100 ; z / \delta<0.15$ ) is populated by very long streamwise organized motions. Here $z$ is the wall-normal distance, $\delta$ is boundary layer thickness and the + superscript means normalization with wall variables $\left(U_{\tau}\right.$ and $v / U_{\tau}$, where $U_{\tau}$ is the friction velocity and $v$ is the kinematic viscosity). Hutchins and Marusic [2] found a regime of very long meandering positive and negative streamwise velocity fluctuations, termed "superstructures", to exist in the $\log$ and lower wake regions of turbulent boundary layers. They are referred to

I. Marusic $(\bowtie) \cdot$ N. Hutchins

Walter Bassett Aerodynamics Laboratory, Department of Mechanical Engineering,

University of Melbourne, Victoria 3010, Australia

e-mail: imarusic@unimelb.edu.au 
as superstructures because of their very long streamwise extent and because they account for a major proportion of the total Reynolds shear stress in the boundary layer [6]. These large organized motions are likely related to the VLSM structures discussed by Adrian and coworkers [1, 5]. Hutchins and Marusic [2] reported measurements made with a spanwise rake of ten hot-wires in two separate facilities (spanning more than a decade of $R e_{\tau}$ ), and these were compared with existing PIV and DNS results. In all cases, evidence of a large-scale stripiness in the streamwise velocity fluctuations was found. The length of these regions commonly reached $20 \delta$. Similar length-scales have been previously reported for pipes and DNS channel flows [7-9]. The large structures were also found to maintain a presence or footprint in the near-wall region, seeming to modulate or influence the near-wall cycle. This is discussed by Hutchins and Marusic [10], who used velocity decomposition techniques to show that the superstructures are not merely superimposed as a low-wavenumber mean shift onto the near-wall region, but rather appear to actively modulate the production of near-wall scales. As part of this process, these large scales also seem to play a crucial role in redistributing fine-scale turbulent motions throughout the boundary layer.

In this paper we consider the log region structure further and pay particular attention to two-point velocity correlations. These correlations are fundamental quantities giving insights into the statistically significant motions or structures in the flow. More importantly, we consider how these statistics and others scale and change with increasing Reynolds number. To do this we use three separate experimental facilities and three different measurement techniques to obtain a Reynolds number range of nominally $10^{3} \leq R e_{\tau} \leq 10^{6}$, where $R e_{\tau}=\delta U_{\tau} / \nu$. The lowest of these Reynolds numbers are typical of small-scale or low-speed laboratory facilities and are obtainable by DNS (direct numerical simulation), while $R e_{\tau}=O\left(10^{6}\right)$ is typical of the Reynolds number that may be encountered on the fuselage of large aircraft or on the hulls of submarines or ships.

\section{Experimental Facilities}

The experiments were conducted in three different facilities: a low-speed wind tunnel at the University of Minnesota $\left(R e_{\tau} \approx 1,000\right)$, the high Reynolds number boundary layer wind tunnel at the University of Melbourne $\left(R e_{\tau} \approx 14,000\right)$, and at the SLTEST site on the salt flats of Western Utah $\left(\operatorname{Re}_{\tau} \approx 6 \times 10^{5}\right)$. Specific details of each facility can be found in $[11,12]$ and $[13]$ respectively. The three facilities and measurement techniques are detailed in Fig. 1. As indicated on the figure, throughout this paper $x$, $y$ and $z$ denote the streamwise, spanwise and wall-normal directions respectively.

The lowest Reynolds number experiments where made at the University of Minnesota in an open return suction-type boundary layer wind-tunnel of working section $4.7 \times 1.2 \times 0.3 \mathrm{~m}$ at $R e_{\tau} \approx 1,000$. The measurements consist of stereoscopic PIV measurements made simultaneously on two mutually perpendicular planes (both streamwise/wall-normal, and streamwise/spanwise). Polarization techniques were employed to acquire the PIV data in both planes simultaneously, and image preprocessing techniques were used to improve the quality of data near the line of intersection of the planes. Full details of the measurements and facility are available in [11].

空 Springer 
Fig. 1 Details of three measurement facilities in order of increasing Reynolds number. a Simultaneous combined-plane stereo PIV used in a low Reynolds number wind-tunnel at the University of Minnesota; b spanwise rake of ten hot-wire probes in the high Reynolds number wind-tunnel at the University of Melbourne; c sonicanemometer array deployed in the atmospheric surface layer at SLTEST Utah

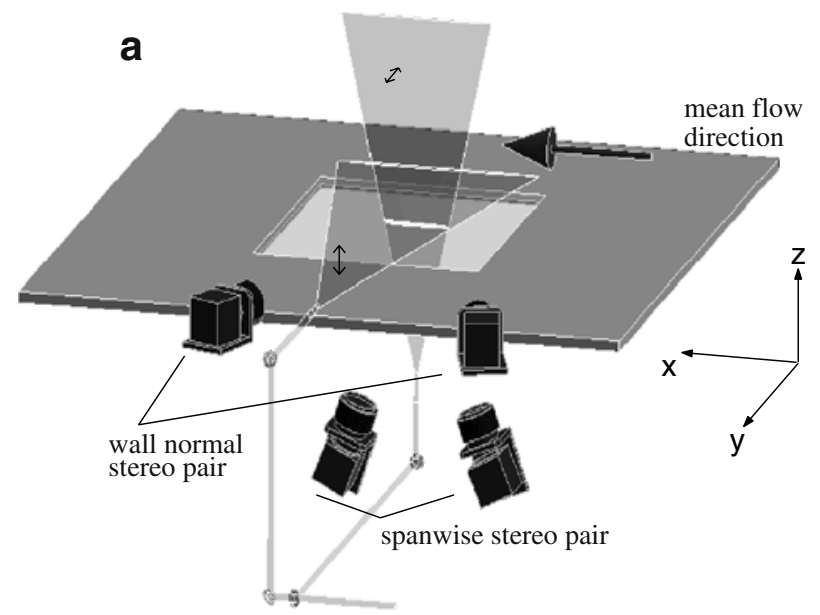

b

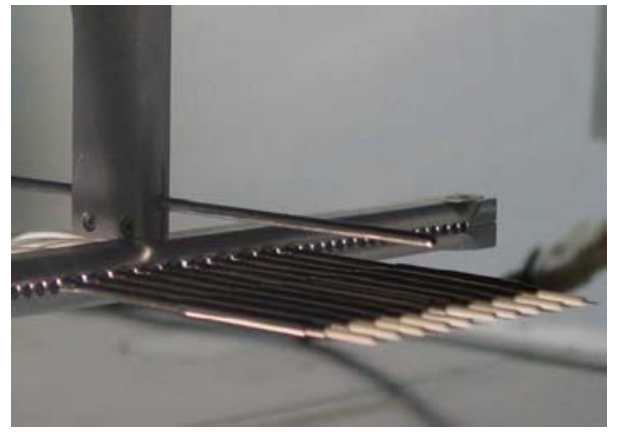

C

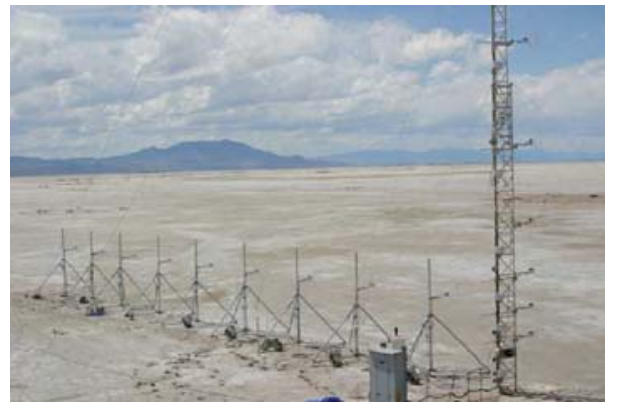

The highest Reynolds number laboratory data were obtained at the University of Melbourne in an open return blower-type wind tunnel with working section $27 \times 2 \times 1 \mathrm{~m}$. This facility can produce Reynolds numbers up to $R e_{\tau}=26,000$ (see $[12,14]$ for details of this facility). In this case the logarithmic region of the turbulent boundary layer was characterised using a spanwise rake of hot-wires. This technique is described in [2]. The hot-wire rake consists of 10 Dantec 55P16 single sensor hotwire probes with a spanwise spacing such that the entire rake measures a spanwise 
domain just greater than one boundary layer thickness. The probes have $1.25-\mathrm{mm}$ long platinum-plated tungsten wire sensing elements of $5 \mu \mathrm{m}$ diameter and are operated in constant temperature mode using an AA Lab Systems AN-1003 with overheat ratio set to 1.8 .

The highest Reynolds number data are from the atmospheric surface layer (ASL). These experiments were conducted at the SLTEST facility, located in the Dugway Proving Grounds, Utah. The unique geography of this site enables measurements to be made in extremely high Reynolds number turbulent boundary layers $\left[\operatorname{Re}_{\tau} \approx\right.$ $O\left(10^{6}\right)$ ] that have developed over nominally $100 \mathrm{~km}$ of low-surface-roughness featureless salt-flat. Full details of the facility are available in [15, 16] and [13]. These atmospheric measurements are essentially a scaled-up version of the hot-wire rake experiments, with wall-normal position and spanwise separation scaled on the expected boundary layer thickness ( $\delta$ is estimated at $60 \mathrm{~m}$ ). A spanwise array of ten sonic anemometers (Campbell Scientific CSAT3) are deployed on tripods mounted $2.14 \mathrm{~m}$ above the desert floor and spaced $3 \mathrm{~m}$ apart in the spanwise direction. At one end of the spanwise array is located an additional wall-normal array of 9 logarithmically spaced sonic anemometers (from $z=1.4$ to $25.7 \mathrm{~m}$ ). A photograph of this array deployed at SLTEST is included in Fig. 1c.

\section{Results and Discussion}

The Utah experiments were conducted under nominally neutrally buoyant conditions and the velocity profiles obtained were as expected from a shear-driven wallbounded flow. We consider here one hour of data taken from a period of neutral buoyancy and steady wind conditions during the early hours of June 2nd, 2005. Figure 2 collates the mean velocity profiles from all three experiments. Here the friction velocity, $U_{\tau}$, was obtained using a Clauser chart $(\kappa=0.41, A=5.0)$ for the laboratory experiments, while for the Utah measurements it was obtained from the Reynolds shear stress from the lowest sonic anemometer [13]. The error bars for the SLTEST data indicate the confidence level for this estimate of $U_{\tau}$. Since the Clauser chart technique was used nothing can be said about the universality of the

Fig. 2 Inner-scaled mean velocity profile (star) combined-plane PIV data, $R e_{\tau} \approx 1,000 ;$ (box, triangle) hot-wire data from Melbourne tunnel, $R e_{\tau} \approx 7,300$ and 20,000; (bullet) SLTEST data $R e_{\tau} \mathrm{O}\left(10^{6}\right)$. Lines show $U^{+}=z^{+} ;($broken line $)$ $U^{+}=\frac{1}{\kappa} \ln \left(z^{+}\right)+A$

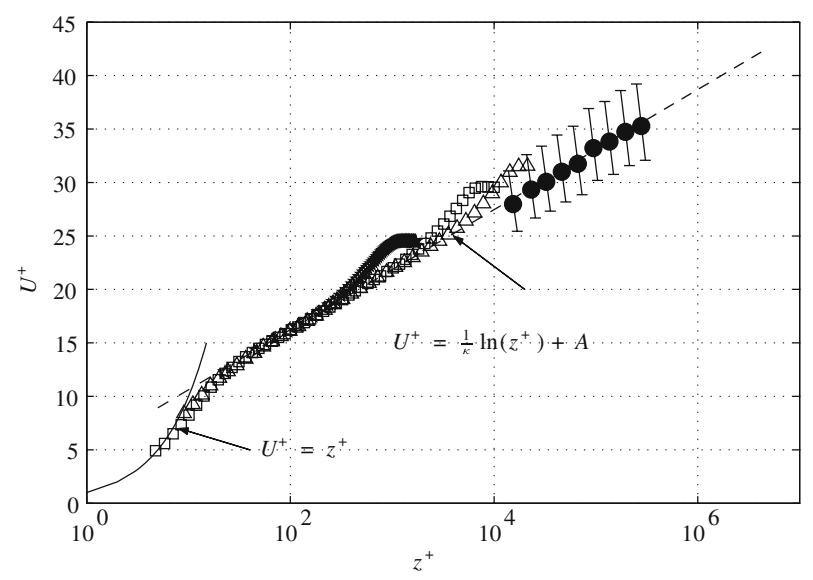


logarithmic region constants. However, all three Reynolds number flows show strong support for a logarithmic profile. Turbulence statistics (not shown here) also agree well with the expected trends as described by Marusic and Kunkel [17].

Comparisons of the two-point correlations of the fluctuating streamwise velocity $\left(R_{u u}\right)$ in the log layer are shown in Fig. 3, for the hot-wire rake and SLTEST experiments. Plot $(a)$ shows the correlations in the streamwise direction at $\Delta y=0$
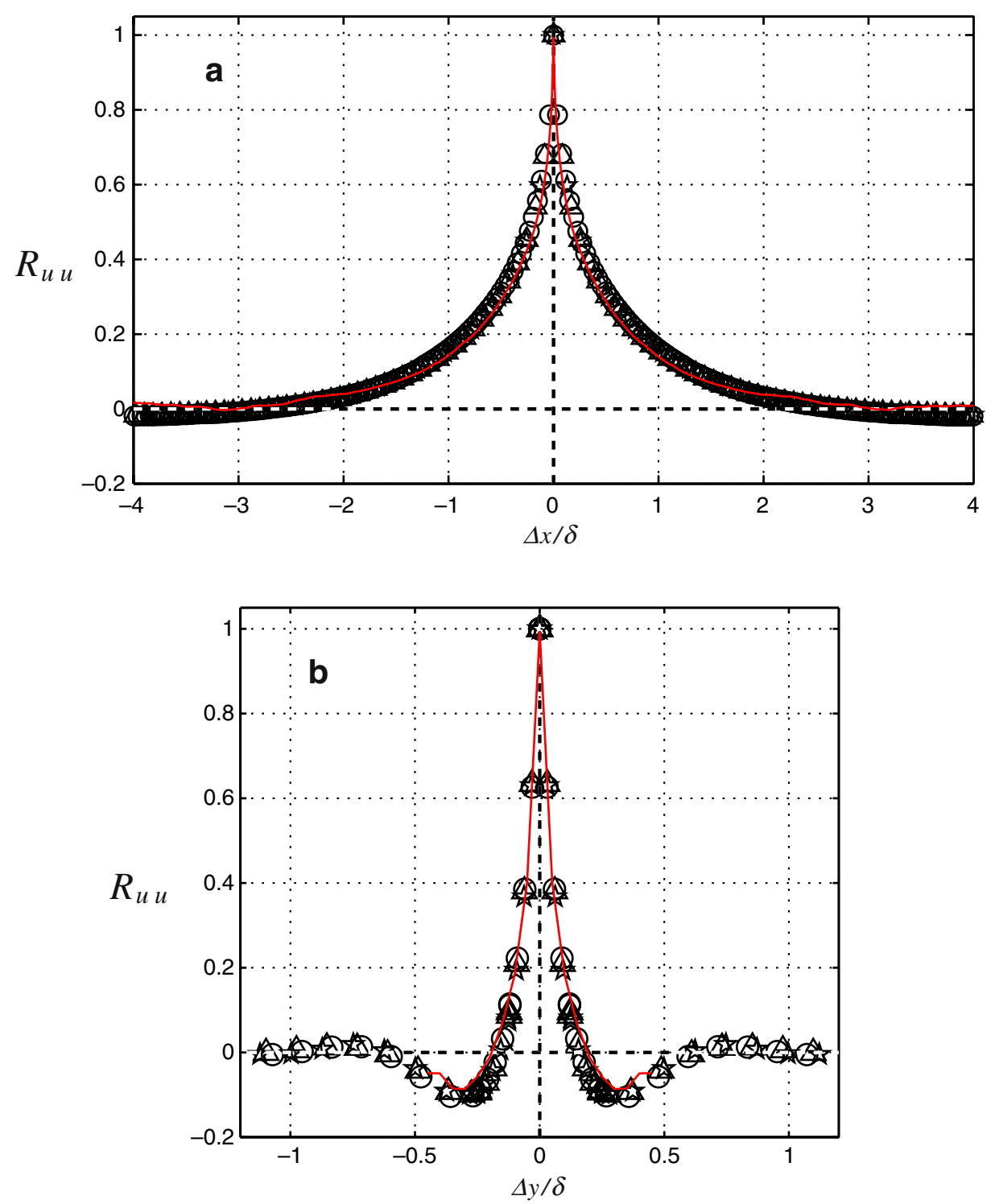

Fig. 3 Two-point correlations of the streamwise velocity fluctuation $R_{u u}$ calculated from the spanwise array at $z_{\mathrm{ref}} / \delta \approx 0.05$. Symbols show rake data [2] (circle) $R e_{\tau}=7,611 ;$ (triangle) $R e_{\tau}=14,379 ;$ (star) $R e_{\tau}=19,956$. Solid line is Utah data (at $\left.z / \delta \approx 0.036\right)$ 
a Combined-plane PIV $R e_{\tau} \approx 1000$

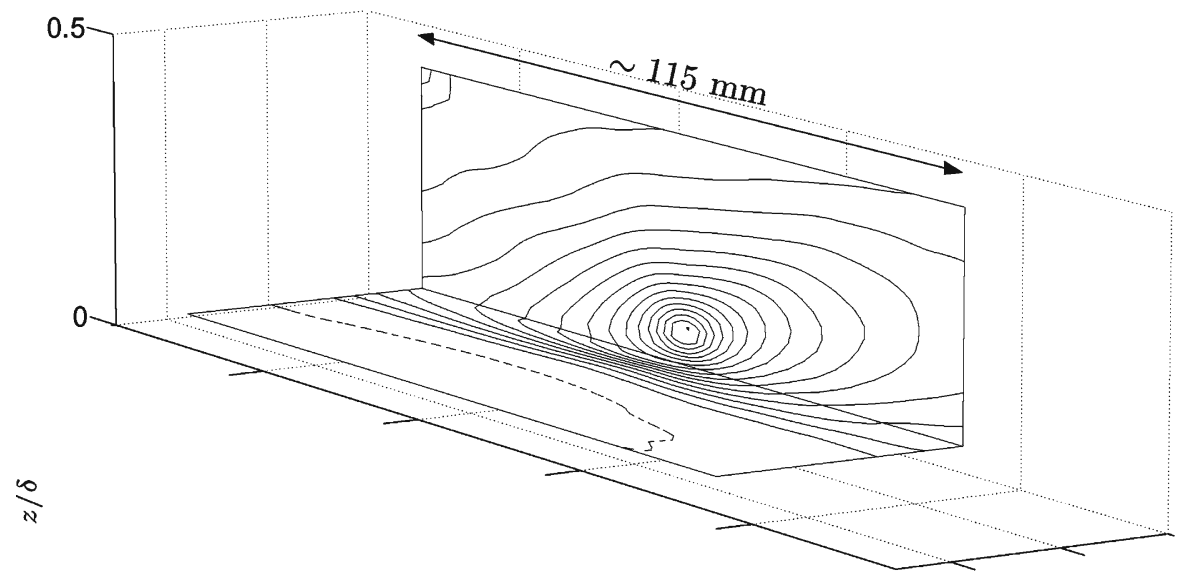

b SLTEST sonic anemometry $R e_{\tau} \approx 6.6 \times 10^{5}$

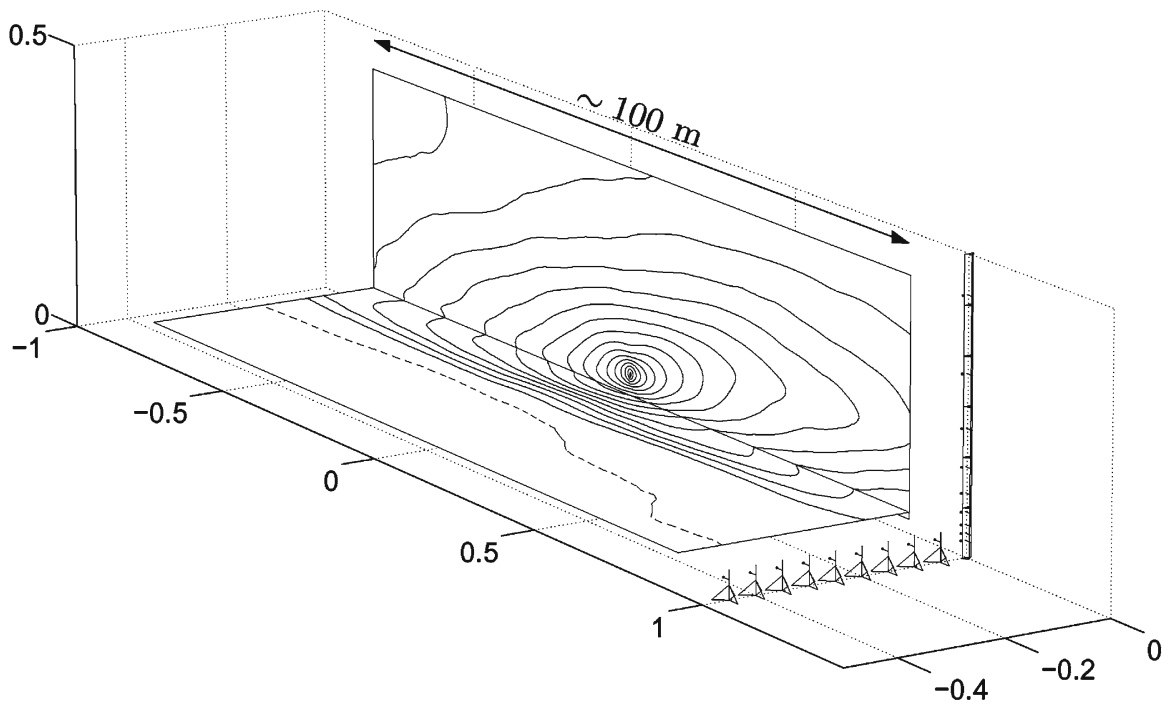

$\Delta x / \delta$

Fig. 4 Comparison of $R_{u u}$ contours from two very different facilities a Combined-plane PIV from low Reynolds number boundary-layer wind-tunnel facility $R e_{\tau} \approx 1,000, z_{\text {ref }}=0.087 \delta ; \mathbf{b}$ atmospheric surface layer at SLTEST, sonic anemometer rake data at $R e_{\tau} \approx 6.6 \times 10^{5}, z_{\text {ref }}=0.071 \delta$. Contour levels are from $R_{u u}=-0.06$ to 0.96 in increments of 0.06. Solid lines show positive contours and dashed show negative. A schematic representation of the measurement array (spanning $27 \mathrm{~m}$ across and $25.7 \mathrm{~m}$ high) is included for reference 
(this is the autocorrelation). The streamwise ordinate in these figures is recovered using Taylor's hypothesis, taking a convection velocity based on the mean across the array. Plot $(b)$ shows the spanwise variation of $R_{u u}$. The symbols in Fig. 3 are hotwire rake data for $R e_{\tau}=7,610-19,960$. The solid line shows the SLTEST data at $R e_{\tau} \approx O\left(10^{6}\right)$. Here we see that outer scaling $(\delta)$ holds remarkably well over the three orders of magnitude change in $R e_{\tau}$ for a nominally fixed $z / \delta$. In attempting to present the SLTEST data on these figures, we are faced with the usual problem that in the atmosphere we do not have a precise value for the boundary layer thickness $\delta$. However, the solid lines indicate that if we assume a value for $\delta$ of $60 \mathrm{~m}$, the two-point correlations collapse well with the existing laboratory results. This estimate of $\delta$ also gives consistent behaviour for other statistics such as the turbulence intensities and spectra. Also, it is noted that $\delta=60 \mathrm{~m}$ gives $z / \delta$ of 0.036 for the atmospheric results, which is not directly comparable with the laboratory data (for which $z / \delta=0.05$ ). However, a comparison of the $R_{u u}$ results from [2] for varying $z / \delta$ would suggest that there should be very little change between the curves at $z / \delta=0.036$ and 0.05 . (The variation of the correlation width across the log layer is linear with $z / \delta$.) It should also be cautioned that the advection lengths (in terms of boundary layer thicknesses) are very low for the SLTEST data. Even with one hour of steady flow conditions, the advection length is only approximately $\mathrm{O}(330 \delta)$ (assuming $\delta \approx 60 \mathrm{~m}$ ). By comparison, the velocity signals for the $R e_{\tau}=19,956$ data shown in Fig. 3 have advection lengths of over $37,000 \delta$. This is a good cautionary indication of the difficulties faced in obtaining converged statistics at SLTEST (the same level of convergence for the atmospheric data would require $>100 \mathrm{~h}$ of stable data).

Regardless, from Fig. 3, it is clear that the streamwise and spanwise correlation profiles are almost identical when scaled with boundary layer thickness over the three orders of magnitude change in Reynolds number. This remarkable degree of collapse suggests that the largest scale structures in the turbulent boundary layer scale on (something close to) boundary layer thickness up to $R e_{\tau} \approx \mathrm{O}\left(10^{6}\right)$.

Corresponding two-point correlation plots $\left(R_{u u}\right)$ for the two orthogonal planes are shown in Fig. 4 for the laboratory PIV and Utah sonic array studies. Again, the remarkable quantitative similarity between these planar correlations strongly support outer $(\delta)$ scaling. It is noted that $\delta$ changed significantly between these experiments (by almost three orders of magnitude); being nominally $0.07 \mathrm{~m}$, and $60 \mathrm{~m}$ for the Minnesota and Utah experiments respectively. Hence, in dimensional terms, the lower plot $(b)$ depicts atmospheric scale structures that are close to 1,000 times larger than those measured in a laboratory (as shown above in plot $a$ ). To the best of our knowledge these are the first such detailed comparisons of correlation structures over such a large range of Reynolds numbers.

\section{Instantaneous Structure}

Apart from statistical quantities, such as correlations, it is useful to consider the instantaneous form of the very large features in the log region. Figure 5 shows examples of instantaneous fields of negative streamwise fluctuating velocity. Figure 5 a shows an example segment from a hot-wire rake at $z / \delta=0.10$. Here the instantaneous spanwise variation of $u$ is projected in time using Taylor's hypothesis to produce a planar view. A very long meandering feature appears to snake through the 
Fig. 5 a Example hot-wire rake $u$ signal at $z / \delta=0.10$ for $R e_{\tau}=14,380$. b Typical combined-plane PIV frame for comparison at $R e_{\tau}=1,100$, $z / \delta=0.087$. c Example signal from sonic anemometer rake in the ASL. The $x$ axes of $\mathbf{b}$ and $\mathbf{c}$ are reconstructed using Taylor's hypothesis and a convection velocity based on the local mean. Shading shows only negative $u$ fluctuations (see gray-scale)
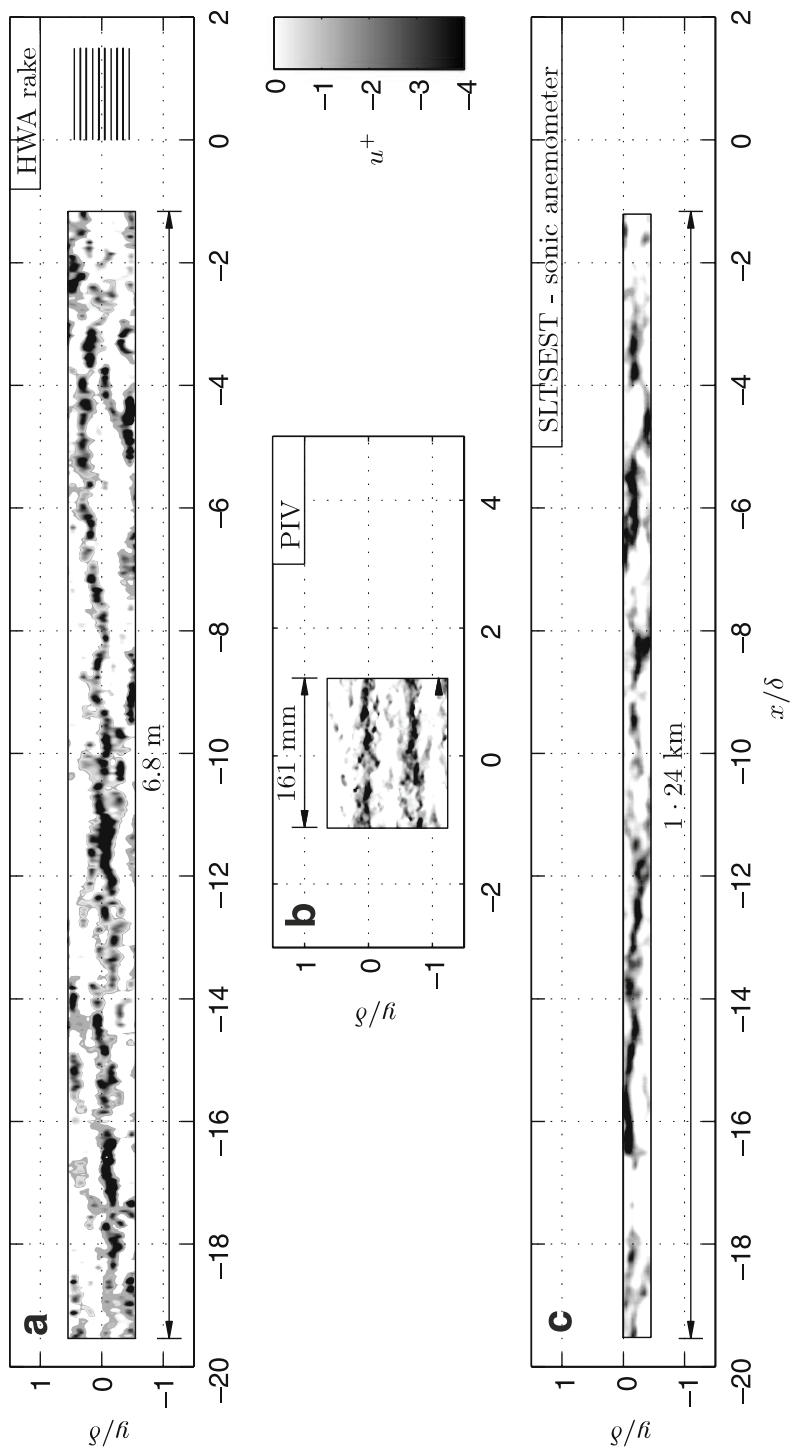

measurement domain for over $18 \delta$. For the Reynolds number shown $\left(R e_{\tau}=14,380\right)$ this amounts to a physical length of over $6 \mathrm{~m}$. This implies that the log region stripiness previously observed from PIV data $[6,18]$ is actually a snapshot of a far larger structure (see the PIV comparison in Fig. 5b). Furthermore, although the lowspeed region in Fig. 5a is clearly very long, the meandering tendency will tend to mask its true length from single point statistics. This meandering may explain why correlations, such as those shown in Fig. 3a, and the location of the peak in the premultiplied spectra $k_{x} \Phi_{u u}$ have consistently indicated shorter length-scales $O(6 \delta)$ for turbulent boundary layers. 


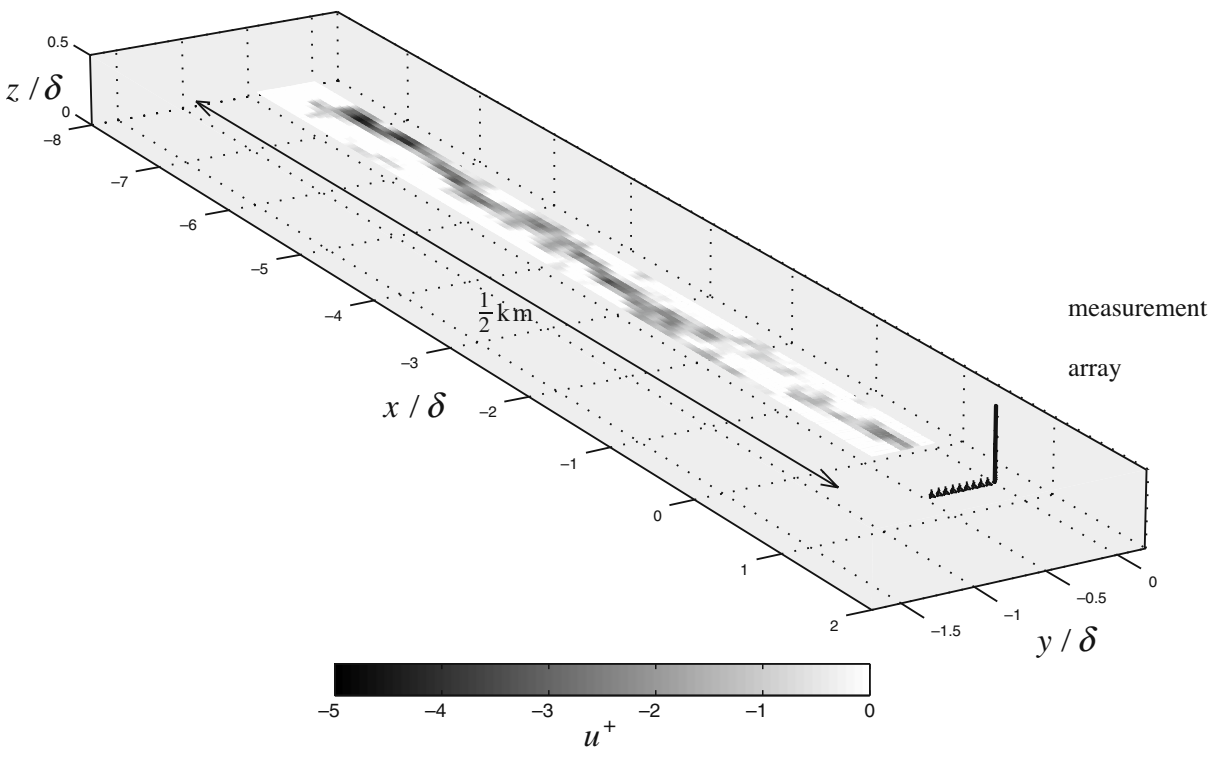

Fig. 6 Example signal across the spanwise array of sonic anemometers at SLTEST, $z / \delta \approx 0.037$. The $x$-axis is reconstructed using Taylor's hypothesis and a convection velocity based on the local mean, $\bar{U}=5.46 \mathrm{~ms}^{-1}$. Shading shows only negative $u$ fluctuations, as indicated on the gray-scale. (Figure taken from [2])

Figure 5c shows the corresponding instantaneous streamwise velocity fluctuations for the Utah measurements. Here a 230 second trace across the spanwise array is shown. A larger and zoomed-in view is shown in Fig. 6, where a schematic of the measurement array is shown to scale to give some sense of proportion. The streamwise ordinate is reconstructed using Taylor's hypothesis, in exactly the same way as for Fig. 5a. It is immediately obvious that similar very long meandering features inhabit the log region of the atmospheric surface layer. It is difficult to ascertain their full streamwise extent given the meandering tendency of these largescale features and the limited spanwise field of view (the spanwise width of the sonic anemometer array is only $0.5 \delta$ and the sample length is also somewhat limited). However, it is clear that these large structures do persist at high Reynolds number and appear to be universally present in the logarithmic region of nominally zero pressure gradient turbulent boundary layers. An important question then arises as to the influence of these motions, for example, in regard to transport mechanisms, and whether or not these influences change with Reynolds number. A closer investigation of the induced average motions is considered in the following section.

\section{An Associated Large-Scale Vortical Structure}

Through closer analysis of the two-point cross-correlations from the combined-plane PIV experiments, it is evident that in addition to the meandering streaks of low and high-speed $u$ fluctuation in the log region, the superstructure is also present as a 
large-scale $v$ and $w$ fluctuation that looks very much like an accompanying largescale roll-mode of counter-rotating vortices.

Figure 7 shows planar views of $R_{u u}, R_{u v}$ and $R_{u w}$ from the PIV experiments. For Fig. 4 the reference point for correlation was located in the vertical plane of the orthogonally arranged PIV pair. To produce the correlation volume shown in Fig. 7 the reference point is located at a given spanwise location $\left(y_{\text {ref }}\right)$ within the horizontal plane. This yields the accompanying vertical correlation plane $\Delta y_{\text {ref }}$ to the side of the reference point (since the vertical PIV plane intersects the horizontal at $y=0)$. By repeating this process at all values of $y_{\text {ref }}$ a full volume of crosscorrelation coefficients can be constructed. Figure 7 shows slices through this volume. The horizontal $(x-y)$ plane is at $z / \delta=0.02$, the cross-plane $(y-z)$ is at $\Delta x=0$ and the vertical plane $(x-z)$ is at $\Delta y / \delta=0.35$. These planes are chosen for clarity and to illustrate key features of the correlation maps.

Fig. 7 Two-point cross-correlations of streamwise velocity fluctuation with a streamwise velocity fluctuation $R_{u u}$; b spanwise velocity fluctuation $R_{u v}$; c wall-normal velocity fluctuation $R_{u w}$. Solid contours show positive correlations (from 0.06 to 0.96 in increments of 0.06 for $R_{u u}$ and 0.01 to 0.08 increments of 0.01 for $R_{u v}$ and $\left.R_{u w}\right)$. Dashed contours show negative correlations (from -0.12 to -0.06 in increments of 0.06 for $R_{u u}$ and -0.08 to -0.01 increments of 0.01 for $R_{u v}$ and $\left.R_{u w}\right)$. Reference point is shown by the plus sign

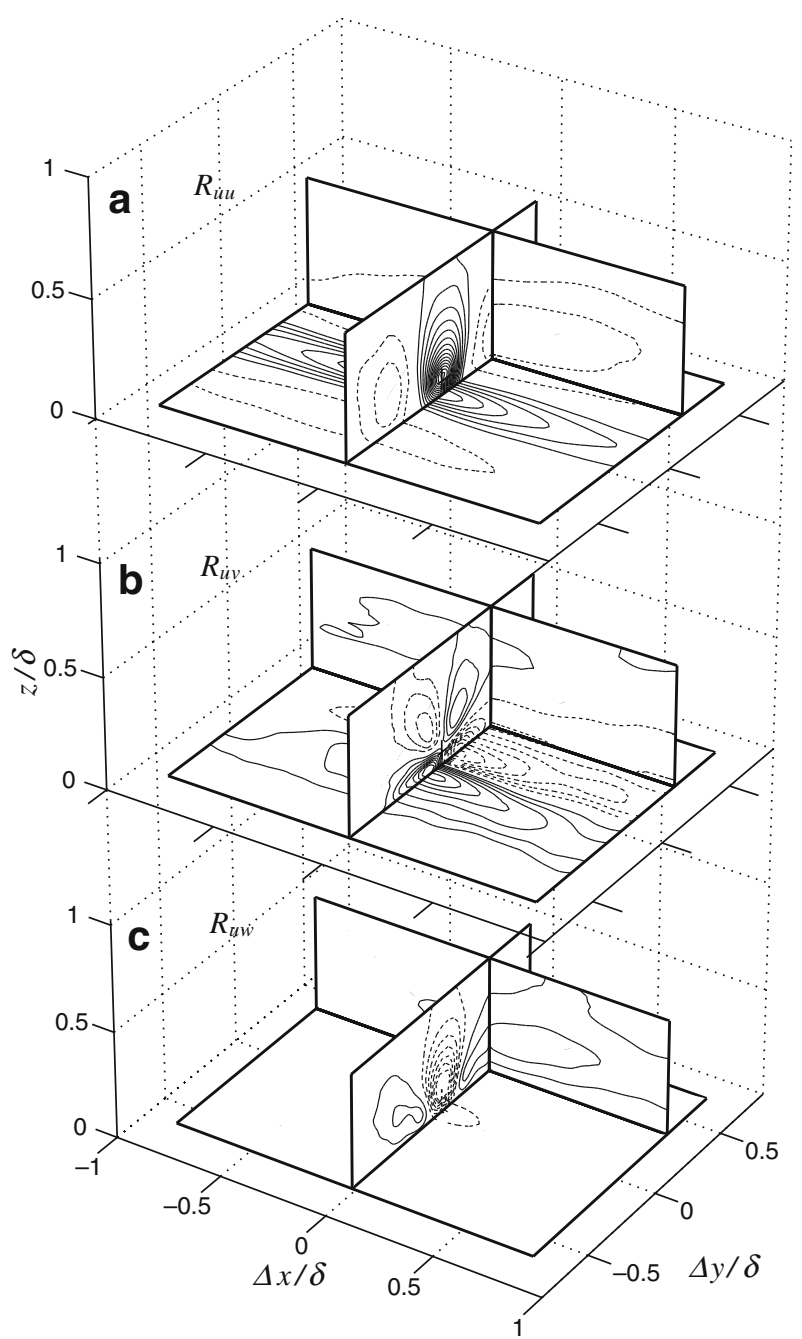


Considering first the streamwise-streamwise correlations $\left(R_{u u}\right)$ of Fig. 7a. A $u$ fluctuation at the reference position (marked by the + symbol) is accompanied by a highly elongated region of positive correlation, flanked on either side in the spanwise direction by anti-correlated behaviour. This would indicate that $u$ fluctuations in the log region are highly elongated and flanked by opposite signed fluctuation in $y$ (as evidenced by the instantaneous realizations of Figs. 5 and 6).

More interesting for this discussion are the correlations of $u$ fluctuations with the remaining two velocity components. Figure $7 \mathrm{~b}$ shows the streamwise-spanwise correlation $\left(R_{u v}\right)$ contours. The arrangement of correlated and anti-correlated behaviour indicates that a negative $u$ fluctuation in the log region, will be accompanied by converging spanwise flow close to the wall, and a diverging spanwise flow above the reference point (and vice versa for a positive $u$ fluctuation). The corresponding streamwise-wall-normal $\left(R_{u w}\right)$ contours of Fig. $7 \mathrm{c}$ show that the negative $u$ fluctuation will be accompanied by an upwash at the reference point, with flanking downwash regions to either side in $y$. Together these correlations indicate that the superstructure events of long meandering regions of positive and negative $u$ fluctuation will be accompanied by counter-rotating roll-modes. This is clarified in Fig. 8, which shows a vector map constructed from $-R_{u v}$ and $-R_{u w}$ on the $y-z$ plane at $\Delta x=0$. This illustrates the cross-plane flow that will (statistically) accompany a low-speed superstructure type event. Such a vector map is approximately equivalent to the linear stochastic estimate (see [19]) where the condition vector is a negative signed $u$ event at the reference point. Clearly a counter-rotating roll-mode will accompany the elongated low and high $u$ momentum regions of the superstructure (with common-flow-up located at the low-speed streak and common-flow down at the high-speed). Such large-scale roll-modes have been previously observed in turbulent boundary layers, most recently by del Álamo et al. [20]. Kim and Adrian [1] have previously suggested that the very long $u$ modes present in pipe flows

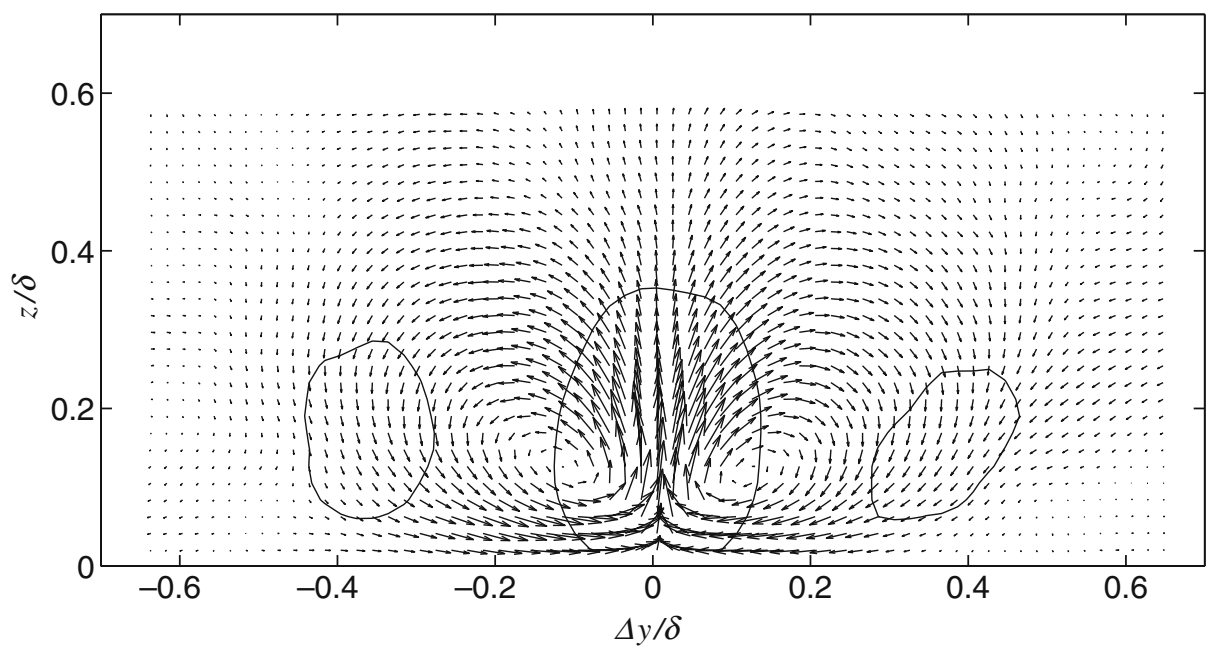

Fig. 8 Vector plot constructed from $-R_{u v}$ and $-R_{u w}$ cross-correlations on the spanwise/wall-normal plane at $\Delta x=0$ (the cross-plane shown in Fig. 7). The contours show $R_{u и}$ correlations at (solid) $R_{u u}=0.18$ and (dashed) $R_{u u}=-0.12 . z_{\text {ref }}=0.087 \delta$ 
may in fact be agglomerations of hairpin packets (themselves agglomerations of individual attached eddy type events, see [21, 22]). Recently, Balakumar and Adrian [23] have observed that 'the smoothed field of a concatenation of misaligned hairpin packets would look like a pair of meandering counter-rotating streamwise vortices, whose diameters would be of the order of the height and width of the packets.' Certainly there is ample evidence in the literature to suggest a complex clustering of small-scale vortex structures around the elongated $u$ fluctuations in the log region, $[6,11,18,21,24-28]$. In a mean sense at least, and from the planar views afforded by PIV, such a clustering seems to be fairly well described by packets of attached hairpin type eddies, although instantaneous volumetric realizations (primarily from DNS) indicate a more complex clustering. Whether or not the large-scale rollmodes shown in Fig. 8 can be attributed to the jittered effect of these small-scale vortices (as suggested by Balakumar and Adrian [23]), or whether they are some separate instability associated with the superstructure (i.e. [29]) should at this stage be considered an open question.

Also of interest in Fig. 7 are the correlation contours on the wall-parallel plane at $z / \delta=0.02$. This effectively shows the near-wall contribution due to a large-scale $u$ event in the log region. Note that whilst the $R_{u u}$ and $R_{u v}$ components show a largescale contribution at $z / \delta=0.02$, the wall-normal component $R_{u w}$ exhibits a much more spatially compact presence close to the wall. This is consistent with Townsend's [30] notion of inactive motions, whereby large-scale eddies primarily produce only wall-parallel motions at the wall (due to the blocking effect of the wall). For vortex based models, one can interpret this blocking as the image vortices at the wall. Energy spectra from DNS of channel flows [4] have confirmed that the spanwise fluctuating component contains energy at large outer-scaled wave-numbers close to the wall (whilst the wall-normal component does not). This is as predicted by the attached eddy hypothesis [30-32], which predicts a large-scale contribution to $u$ and $v$ near to the wall, but not to $w$ or the Reynolds shear stress $-\overline{u w}$.

\section{A Modulating Influence at the Wall}

The terms 'active' and 'inactive' motions can be misleading when interpreted literally. Here we use 'inactive' only in Townsend's [30] original sense; when referring to motions that are predominantly wall-parallel. Though the near-wall signature of the superstructure-type events are largely of this wall-parallel category, they nonetheless seem to exert considerable influence onto the near-wall cycle. Through decomposition of hot-wire signals in a turbulent boundary layer at $R e_{\tau} \approx 7,300$, the authors have recently established [10] that the superstructure events modulate the near-wall cycle (in a manner akin to amplitude modulation). By studying small viscous-scaled fluctuations within large outer-scale events, it is apparent that the low momentum footprint of the superstructure is accompanied by a reduction in smallscale fluctuations near the wall. Conversely, the high-speed superstructure footprint

Fig. 9 DNS data a streamwise velocity fluctuations in the $\log$ region at $z^{+}=100$; b as above but with addition of Guassian filtered contour field at $u_{f}^{+}=-0.5$; c zoomed view of region marked in $\mathbf{b}$;

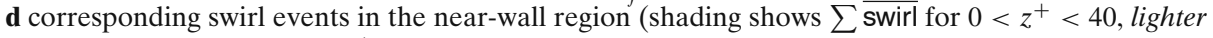
shading shows increased swirl)

글 Springer 

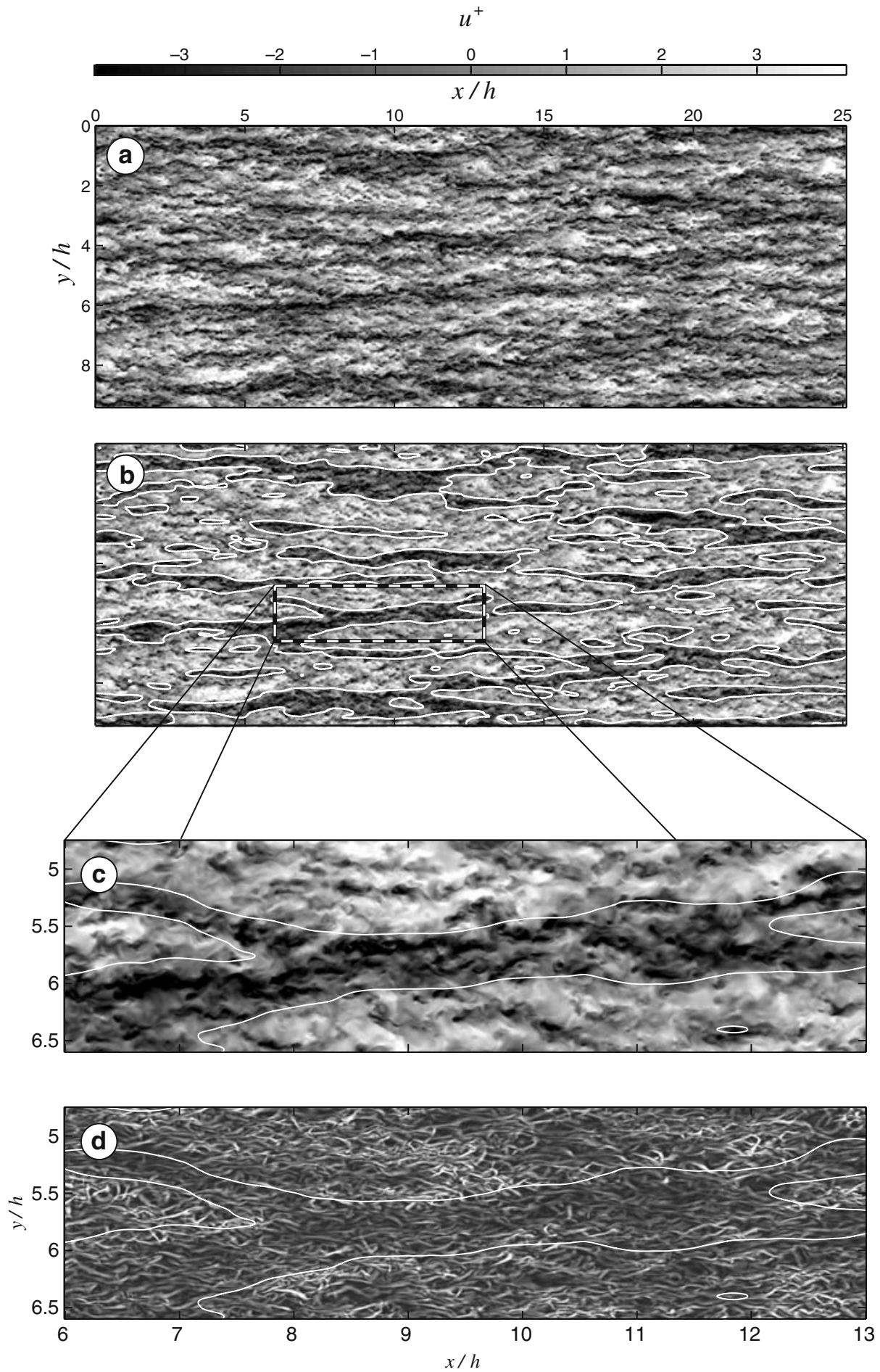
(which flanks the low-speed) is accompanied by increased small-scale activity close to the wall. These findings are consistent with previous studies of Bandyopadhyay and Hussain [33] and Rao et al. [34] that show that large outer-scaled structures are active in rearranging and interacting with the near-wall structure.

One obvious restriction of hot-wire studies is the limited spatial area that can be simultaneously measured. Even when multiple probes are employed, we are often restricted to single component velocity measurements along a single spatial line. Direct numerical simulation (DNS) overcomes this by providing full volumetric three-dimensional data, and while the Reynolds numbers achievable in DNS are still limited these data sets are very useful particularly if used in combination with other higher Re experimental studies. Here we use the DNS database of del Álamo et al. [3] to consider the influence of the large log region structures on the near-wall viscous region. Hutchins and Marusic [10] used the same data set to show that the above mentioned modulation effect was evident in fluctuating $v, w$ and $u w$ components, in addition to the $u$ modulation identified in hot-wire experiments.

As an illustration of this phenomena, Fig. 9a shows a streamwise-spanwise plane of $u$ fluctuations in the log region of the DNS channel flow of [3]. Note the large numerical domain ( $8 \pi h$ in $x$ and $3 \pi h$ in $y$, where $h$ is the channel half height). Highly elongated large-scale motions are visible in this velocity field, primarily as elongated dark regions of shading. These low-speed superstructure events are highlighted in Fig. 9b by white iso-contours drawn through the Gaussian filtered velocity field at $u_{f}^{+}=-0.5$ (filter size $\frac{h}{2} \times \frac{h}{2}$ ). Figure 9c shows a zoomed view of such an event. To highlight small-scale activity at the wall, the instantaneous velocity gradient tensor is used to calculate swirl. This has been shown to be a useful marker of compact swirling motions (see [35]). Figure 9d shows the summation of swirl from $0<z^{+}<40$ for the same zoomed area. In general, this near-wall region exhibits a predominance of quasi-streamwise small-scale vortices, typical of the nearwall cycle. However, if we focus attention on the region beneath the low-speed superstructure event, it is clear that the region encapsulated by the white contour exhibits reduced swirling activity (a darker overall shading). Accordingly, the highspeed footprint of the superstructure (to either side of the contour) is characterized by increased swirl activity. This would seem to reaffirm the findings of [10], namely that the near-wall large-scale inactive motions due to the superstructure, appear to amplify (or attenuate, depending on phase) the small-scale activity at the wall. As Reynolds number increases the separation in scale between the superstructure and the near-wall cycle will go nominally as $6 R e_{\tau} / 1,000[2,10]$, resulting in increasingly inhomogeneous distributions of near-wall scales under the large-scale modulating influence of the superstructure.

\section{Conclusions and Summary}

We have presented results from three different experimental studies, and used these data to investigate the large scale structure of the logarithmic region of wall turbulence. The measurements span over three decades of Reynolds number and were conducted in three separate facilities: two laboratory scale facilities and the atmospheric surface layer at the SLTEST facility in Utah. Three different measurement techniques were used: PIV, hot-wire and sonic anemometry. 
Two-point correlation statistics at a given $z / \delta$ were found to agree well over all Reynolds numbers when scaled with the outer length-scale $\delta$. These correlation maps are consistent with very large scale features in the logarithmic region, which we refer to as "superstructures" and which, in instantaneous realizations, appear as a marked stripiness in the fluctuating streamwise velocity with very long length-scales and accompanied by a spanwise meandering. These are consistent with what have been termed VLSM (very large scale motions) by Kim and Adrian [1]. Evidence for these large structures is found in all three facilities, including the atmospheric surface layer. Verification of the existence of "superstructure" type events in the atmospheric surface layer raises many challenging questions for structural based turbulence models. For example, at laboratory scale, correlation maps such as those shown in Figs. 3 and 4 have in the past been associated with the statistical imprint of hairpin packet structures and wall-up type structural models (models where vortex structures grow upwards from the wall). Such models are challenged by these atmospheric results, where the enormous degree of scale separation requires viscous scale motions (100 viscous wall units for the Utah data is approximately $10 \mathrm{~mm}$ ) to grow into or give rise to structures on the kilometer scale.

Results are also presented using a database from a DNS of channel flow to show that the large-scale superstructure events have a significant interaction with the near-wall region, seeming to modulate the small-scale activity at the wall. Similar behaviour was verified in experimental data in [10]. Results presented in [2] suggest that this modulating influence will intensify with increasing Reynolds number.

Acknowledgements The authors wish to gratefully acknowledge the support of the Australian Research Council, through the Federation Fellowship program and grant DP-0663499, the US National Science Foundation though grant CTS-0324898, and the David and Lucile Packard Foundation. We also wish to thank Professor Joe Klewicki for his hospitality at the SLTEST site in Utah.

\section{References}

1. Kim, K.C., Adrian, R.J.: Very large-scale motion in the outer layer. Phys. Fluids 11(2), 417-422 (1999)

2. Hutchins, N., Marusic, I.: Evidence of very long meandering streamwise structures in the logarithmic region of turbulent boundary layers. J. Fluid Mech. 579, 1-28 (2007)

3. del Álamo, J.C., Jiménez, J., Zandonade, P., Moser, R.D.: Scaling of the energy spectra of turbulent channels. J. Fluid Mech. 500, 135-144 (2004)

4. Hoyas, S., Jiménez, J.: Scaling of the velocity fluctuations in turbulent channels up to $R e_{\tau}=2003$. Phys. Fluids 18, 011702 (2006)

5. Guala, M., Hommema, S.E., Adrian, R.J.: Large-scale and very-large-scale motions in turbulent pipe flow. J. Fluid Mech. 554, 521-542 (2006)

6. Ganapathisubramani, B., Longmire, E.K., Marusic, I.: Characteristics of vortex packets in turbulent boundary layers. J. Fluid Mech. 478, 35-46 (2003)

7. Kim, K.C., Adrian, R.: Very large-scale motion in the outer layer. Phys. Fluids 11, 417-422 (1999)

8. Jiménez, J.: The largest scales of turbulent wall flows. In: CTR Annual Research Briefs, Progress in Astronautics and Aeronautics, pp. 943-945. Stanford University (1998)

9. del Álamo, J.C., Jiménez, J.: Spectra of the very large anisotropic scales in turbulent channels. Phys. Fluids 15, 41-44 (2003)

10. Hutchins, N., Marusic, I.: Large-scale influences in near-wall turbulence. Proc. R. Soc. Lond. A 365, 647-664 (2007)

11. Hambleton, W.T., Hutchins, N., Marusic, I.: Multiple plane PIV measurements in a turbulent boundary layer. J. Fluid Mech. 560, 53-64 (2006) 
12. Nickels, T.B., Marusic, I., Hafez, S., Hutchins, N., Chong, M.S.: Some predictions of the attached eddy model for a high Reynolds number boundary layer. Proc. R. Soc. Lond. A 365, 807-822 (2007)

13. Kunkel, G.J., Marusic, I.: Study of the near-wall-turbulent region of the high-Reynolds-number boundary layer using an atmospheric flow. J. Fluid Mech. 548, 375-402 (2006)

14. Nickels, T.B., Marusic, I., Hafez, S.M., Chong, M.S.: Evidence of the $k^{-1}$ law in a highReynolds-number turbulent boundary layer. Phys. Rev. Lett. 95, 074501 (2005)

15. Klewicki, J.C., Metzger, M.M., Kelner, E., Thurlow, E.M.: Viscous sublayer flow visualizations at $R_{\theta} \cong 1500000$. Phys. Fluids 7 (1995)

16. Metzger, M.M., Klewicki, J.C.: A comparative study of near-wall turbulence in high and low Reynolds number boundary layers. Phys. Fluids 13 (2001)

17. Marusic, I., Kunkel, G.J.: Streamwise turbulence intensity formulation for flat-plate boundary layers. Phys. Fluids 15, 2461-2464 (2003)

18. Tomkins, C.D., Adrian, R.J.: Spanwise structure and scale growth in turbulent boundary layers. J. Fluid Mech. 490, 37-74 (2003)

19. Adrian, R.J., Moin, P.: Stochastic estimation of organized turbulent structure: homogeneous shear flow. J. Fluid Mech. 190, 531-559 (1988)

20. del Álamo, J.C., Jiménez, J., Zandonade, P., Moser, R.D.: Self-similar vortex clusters in the turbulent logarithmic region. J. Fluid Mech. 561, 329-358 (2006)

21. Adrian, R.J., Meinhart, C.D., Tomkins, C.D.: Vortex organization in the outer region of the turbulent boundary layer. J. Fluid Mech. 422, 1-54 (2000)

22. Marusic, I.: On the role of large-scale structures in wall turbulence. Phys. Fluids 13(3), 735-743 (2001)

23. Balakumar, B.J., Adrian, R.J.: Large- and very-large scale motions in channel and boundarylayer flows. Proc. R. Soc. Lond. A 365, 665-681 (2007)

24. Christensen, K.T., Adrian, R.J.: Statistical evidence of hairpin vortex packets in wall turbulence. J. Fluid Mech. 431, 433-443 (2001)

25. Tanahashi, M., Kang, S.-J., Miyamoto, T., Shiokawa, S., Miyauchi, T.: Scaling law of fine scale eddies in turbulent channel flows up to $R e_{\tau}=800$. Int. J. Heat Fluid Fl. 25, 331-340 (2004)

26. Kasagi, N., Fukagata, K., Suzuki, Y.: Adaptive control of wall-turbulence for skin friction drag reduction and some consideration for high Reynolds number flows. In: 2nd International Symposium on Seawater Drag Reduction, pp. 17-31. Busan (2005)

27. Hutchins, N., Hambleton, W.T., Marusic, I.: Inclined cross-stream stereo PIV measurements in turbulent boundary layers. J. Fluid Mech. 541, 21-54 (2005)

28. Marusic, I., Hutchins, N.: Experimental study of wall turbulence: implications for control. In: Gad-el-Hak, M. (ed.) Transition and Turbulence Control (2005)

29. del Álamo, J.C., Jiménez, J.: Linear energy amplification in turbulent channels. J. Fluid Mech. 559, 205-213 (2006)

30. Townsend, A.A.: The Structure of Turbulent Shear Flow. Cambridge University Press (1956)

31. Perry, A.E., Chong, M.S.: On the mechanism of wall turbulence. J. Fluid Mech. 119, 173-217 (1982)

32. Perry, A.E., Marusic, I.: A wall wake model for the turbulent structure of boundary layers. Part 1. Extension of the attached eddy hypothesis. J. Fluid Mech. 298, 361-388 (1995)

33. Bandyopadhyay, P.R., Hussain, A.K.M.F.: The coupling between scales in shear flows. Phys. Fluids 27(9), 2221-2228 (1984)

34. Rao, K.N., Narasimha, R., Badri Narayanan, M.A.: The 'bursting' phenomena in a turbulent boundary layer. J. Fluid Mech. 48, 339-352 (1971)

35. Adrian, R.J., Christensen, K.T., Lui, Z.-C.: Analysis and interpretation of instantaneous turbulent velocity fields. Exp. Fluids 29, 275-290 (2000) 